www.nature.com/jhg

\title{
Comprehensive DNA methylation analysis of peripheral blood cells derived from patients with first-episode schizophrenia
}

\author{
Masaki Nishioka ${ }^{1,2}$, Miki Bundo ${ }^{1}$, Shinsuke Koike ${ }^{2}$, Ryu Takizawa ${ }^{2,3}$, Chihiro Kakiuchi ${ }^{2}$ Tsuyoshi Araki ${ }^{4}$, \\ Kiyoto Kasai $^{2}$ and Kazuya Iwamoto ${ }^{1}$
}

Epidemiological studies have revealed that schizophrenia is highly heritable. However, genetic studies have not fully elucidated its etiology. Accumulating evidence suggests that epigenetic alterations may provide an additional explanation of its pathophysiology. We investigated the methylation profiles of DNA in peripheral blood cells from 18 patients with first-episode schizophrenia (FESZ) and from 15 normal controls. Schizophrenia patients were confined to those at the stage of first-episode psychosis. We analyzed the DNA methylation status of $27578 \mathrm{CpG}$ sites by means of the Illumina Infinium HumanMethylation27 BeadChip array. Differentially methylated $\mathrm{CpG}$ sites, which were particularly abundant within $\mathrm{CpG}$ islands, were enriched in genes related to the nuclear lumen, to transcription factor binding, and to nucleotide binding. We also observed differential methylation of the promoters of HTR1E and COMTD1, which are functionally related to genes found to be differentially methylated in schizophrenia patients in previous studies. Our results indicate the site-specific epigenetic alterations in patients with FESZ.

Journal of Human Genetics (2013) 58, 91-97; doi:10.1038/jhg.2012.140; published online 13 December 2012

Keywords: biomarker; $\mathrm{CpG}$ island; epigenetics; first-episode psychosis; gender difference

\section{INTRODUCTION}

Schizophrenia is believed to be a genetic disease; its heritability is estimated to be $\sim 50 \% .{ }^{1}$ Genetic studies, including genome-wide association studies, have identified candidate genes such as major histocompatibility complex genes, ZNF804A, TCF4, NRGN and MIR137. ${ }^{2-4}$ Although these genes may be relevant to the pathophysiology of schizophrenia, low penetrance, low odds ratios or low reproducibility is the limitation of these candidate genes. Accumulating evidence suggests that epigenetic alterations, such as DNA methylation and histone modifications, may provide an additional explanation for the pathophysiology of schizophrenia, because epigenetic profiles may reflect environmental risk factors such as stress and poor nutrition.

In postmortem brains of patients with schizophrenia, DNA methylation alterations in the promoters of GAD, RELN, COMT, SOX10 and other genes have been reported. ${ }^{5-12}$ However, hypermethylation of RELN and COMT was not validated by other groups. ${ }^{13-15}$ Investigations of epigenetic alterations in peripheral tissue samples obtained from schizophrenia patients have revealed hypermethylation of HTR1A in leukocytes, ${ }^{16}$ hypomethylation of HTR2A in saliva, ${ }^{17}$ hypomethylation of $M B-C O M T$ in saliva ${ }^{18}$ and hypermethylation of S-COMT in leukocytes. ${ }^{19}$ Dempster et al. ${ }^{20}$ reported hypermethylation in the ST6GALNAC1 promoter in peripheral blood cells derived from monozygotic twins discordant for major psychosis.

In peripheral tissues, the epigenetic profile is considered to be differed from those of brain cells. However, certain regions of the two cell types may have some common epigenetic characteristics. ${ }^{20-23}$ Unlike brain samples, peripheral tissue samples can be obtained with minimally invasive techniques, permitting the collection of larger samples. Such samples might be useful for longitudinal studies, possibly reflecting the course of the disease.

We hypothesized that epigenetic alterations vary with disease stage and that the DNA of patients at the first-episode psychotic stage should exhibit epigenetic alterations that are closely related to the onset of psychotic symptoms. The inconsistency between previous studies may be partly attributable to the difference of disease stages between patients. We expect that consistent epigenetic alterations can be found in patients by strictly confining the patients to those at a specific disease stage. Therefore, we collected peripheral blood samples from carefully selected patients with first-episode schizophrenia (FESZ) and analyzed comprehensive DNA methylation status

${ }^{1}$ Department of Molecular Psychiatry, Graduate School of Medicine, The University of Tokyo, Tokyo, Japan; ${ }^{2}$ Department of Neuropsychiatry, Graduate School of Medicine, The University of Tokyo, Tokyo, Japan; ${ }^{3}$ Social, Genetic and Developmental Psychiatry Centre, Institute of Psychiatry at The Maudsley, King's College London, University of London, London, UK and ${ }^{4}$ Department of Youth Mental Health, Graduate School of Medicine, The University of Tokyo, Tokyo, Japan

Correspondence: Dr K Iwamoto, Department of Molecular Psychiatry, Graduate School of Medicine, The University of Tokyo, 7-3-1 Hongo, Bunkyo-ku, Tokyo 113-8655, Japan. E-mail: kaziwamoto-tky@umin.ac.jp

Received 24 September 2012; revised 27 October 2012; accepted 5 November 2012; published online 13 December 2012 
for comparison with the DNA methylation status of age-matched healthy controls (CTs).

\section{MATERIALS AND METHODS}

\section{Subjects}

A total of 18 Japanese patients (11 males and 7 females) with FESZ were recruited from the outpatient and inpatient unit of the University of Tokyo Hospital. The mean age of the subjects was $22.8 \pm 4.5$ ( \pm s.d.). We also recruited 15 Japanese age-matched healthy CTs ( 10 males and 5 females; mean age $23.3 \pm 4.0$ ). FESZ patients were defined according to the following criteria: (1) patients who had experienced a first acute psychosis as defined by the Structured Interview for Prodromal Symptoms ${ }^{24}$ and were diagnosed with schizophrenia according to Diagnostic and Statistical Manual of Mental Disorders fourth edition (DSM-IV) after $>6$ months of follow-up, (2) patients who had received antipsychotic medication for $<16$ cumulative weeks and (3) patients whose continuous psychotic symptoms had lasted for $<60$ months. From the CT group, we excluded subjects with a history of psychiatric disease or a family history of an axis I disorder in their first-degree relatives. For both groups, we excluded subjects who had current or past neurological illness, autism spectrum disorder, traumatic brain injury, a history of electroconvulsive therapy or a history of substance abuse. We obtained written informed consent from every subject after complete explanation of this study. This study was conducted in accordance with the Declaration of Helsinki and its Amendments, and was approved by Human Genome, Gene Analysis Research Ethics Committee at the Faculty of Medicine, University of Tokyo.

\section{DNA extraction and sodium bisulfite conversion}

Genomic DNA was extracted from the collected blood samples with a Wizard Genomic DNA Purification Kit (Promega, Madison, WI, USA), according to the manufacturer's instructions. Sodium bisulfite conversion of genomic DNA was performed with an EZ DNA Methylation Kit D500 (Zymo Research, Irvine, CA, USA), according to the manufacturer's instructions.

\section{Illumina Infinium HumanMethylation27 BeadChip Kit}

We obtained DNA methylation profiles from 33 DNA samples (18 FESZ patients and 15 CTs) with an Infinium HumanMethylation27 BeadChip Kit (Illumina, San Diego, CA, USA), according to the manufacturer's protocol. A total of $200 \mathrm{ng}$ of DNA was assayed after bisulfite conversion. The Infinium platform assays $27578 \mathrm{CpG}$ sites covering 14475 genes, most of which are located around transcription start sites. ${ }^{25}$ Among the $27578 \mathrm{CpG}$ sites, 20006 CpG sites (72.5\%) are located inside CpG islands and 7572 CpG sites (27.5\%) are located outside CpG islands. The Infinium platform presents DNA methylation status as a beta-value ranging from 0 (no methylation) to 1 (complete methylation). The beta-value is calculated by means of the following equation:

$$
\text { betavalue }=\text { methylated }_{\text {intensity }} /\left(\operatorname{methylated}_{\text {intensity }}+\text { unmethylated }_{\text {intensity }}\right)
$$

where methylated $\mathrm{d}_{\text {intensity }}$ and unmethylated ${ }_{\text {intensity }}$ are the signal intensities of methylated and unmethylated probes, respectively.

\section{Data analysis}

Principal component analysis was performed with CLC Genomics Workbench 4 (CLC Bio, Katrinebjerg, Denmark). Cluster analysis was performed by the nearest-neighbor method with the amap program package ${ }^{26}$ implemented in the R software environment (ver. 2.13.0; http://www.R-project.org/).

We analyzed the methylation data using Significance Analysis of Microarray (SAM) software ver 3.11. ${ }^{27,28}$ A false discovery rate (FDR) for each probe (local FDR) of $<0.01$ was considered statistically significant. For gender-specific analysis, a local FDR of $<0.05$ was considered significant.

Gene ontology was analyzed with the Database for Annotation, Visualization and Integrated Discovery Bioinformatics Resources ver. 6.7.29,30 Ontology terms that contained $>10$ genes and that had an expression analysis systematic explorer score (a modified Fisher's exact $P$-value) of $<0.001$ were considered significant. For gender-specific analysis, terms that contained $>3$ genes and that had an expression analysis systematic explorer score of $<0.05$ were considered significant, owing to the limited sample size.

Using R, we calculated Pearson's correlations between clinical indicators (positive and negative syndrome scale (PANSS) score, global assessment of functioning (GAF), duration of untreated psychosis (DUP), age at disease onset or age at psychosis onset) and beta-values of the probes that showed significant alterations. A correlation coefficient with an absolute value of $>0.5$ and with $P$-value of $<0.05$ was considered significant.

\section{RESULTS}

Quality control with principal component analysis

Principal component analysis revealed that the data from one FESZ patient had significantly aberrant beta-values (Supplementary Figure 1), possibly due to a complex ethnic background or to experimental artifacts. We excluded this subject and performed subsequent analyses with data from the 17 remaining FESZ patients and the 15 CTs (Table 1). Subsequent principal component analysis of these samples showed no clear separation between the two groups (data not shown).

\section{Unbiased cluster analysis}

We then performed unbiased hierarchical cluster analysis by the nearest-neighbor method. Cluster analysis did not clearly discriminate between the FESZ and CT groups (Figure 1). Cluster analysis with other algorithms also failed to discriminate between the two groups (data not shown).

\section{Global DNA methylation analysis}

The average beta-value for all the CpG sites was significantly lower in the FESZ patients than in the CTs ( $\Delta$ beta-value $=0.005, P=0.0005$, $t$-test). The average beta-value for $\mathrm{CpG}$ sites inside $\mathrm{CpG}$ islands was also significantly lower in the FESZ patients than in the CTs ( $\triangle$ betavalue $=0.008, P=2.08 \times 10^{-5}, t$-test). In contrast, the average betavalue for $\mathrm{CpG}$ sites outside $\mathrm{CpG}$ islands was not statistically different between the two groups (Table 2). Separate analyses of the male and female subjects showed the same tendency (data not shown).

\section{Differentially methylated CpG sites and genes in FESZ patients} SAM revealed that $603 \mathrm{CpG}$ sites had significantly different DNA methylation levels between the FESZ and CT groups (local

Table 1 Clinical data (average \pm s.d.) of the subjects

\begin{tabular}{lcc}
\hline & FESZ patients $(\mathrm{N}=17)$ & CTs (N=15) \\
\hline Male:female & $10: 7$ & $10: 5$ \\
Age at sample collection & $22.8 \pm 4.5$ & $23.3 \pm 4.0$ \\
Age at disease onset & $21.0 \pm 5.0$ & $\mathrm{NA}$ \\
Age at psychosis onset & $21.7 \pm 5.1$ & $\mathrm{NA}$ \\
Age at antipsychotics initiation & $22.6 \pm 4.7$ & $\mathrm{NA}$ \\
DUP (weeks) & $48.6 \pm 87.0$ & $\mathrm{NA}$ \\
Duration of treatment (weeks) & $10.6 \pm 9.1$ & $\mathrm{NA}$ \\
GAF & $40.4 \pm 13.4$ & $\mathrm{NA}$ \\
PANSS-positive & $17.5 \pm 3.4$ & $\mathrm{NA}$ \\
PANSS-negative & $21.8 \pm 8.0$ & $\mathrm{NA}$ \\
PANSS-general & $36.5 \pm 8.9$ & $\mathrm{NA}$ \\
PANSS-total & $75.8 \pm 18.4$ & $\mathrm{NA}$ \\
\hline
\end{tabular}

Abbreviations: DUP, duration of untreated psychosis; FESZ, first-episode schizophrenia; GAF, global assessment of functioning; NA, not applicable; PANSS, positive and negative syndrome scale.

Age at disease onset was defined as the age at which schizophrenic symptoms (including negative symptoms such as social withdrawal) were first observed. Age at psychosis onset was defined as the age at which psychotic symptoms such as auditory hallucination were first observed. 
FDR $<1 \%$ ). Notably, all these top-ranked CpG sites showed hypomethylation in the FESZ patients. As expected, cluster analysis of the methylation levels of these $603 \mathrm{CpG}$ sites successfully discriminated between the FESZ patients and the CTs (Figure 2a). Among the $603 \mathrm{CpG}$ sites, 581 (96.4\%) were located inside CpG islands and $22(3.6 \%)$ were located outside CpG islands. The identified CpG sites were significantly enriched inside CpG islands, compared with the overall $\mathrm{CpG}$ site distribution as determined by means of the Illumina Infinium HumanMethylation27 platform

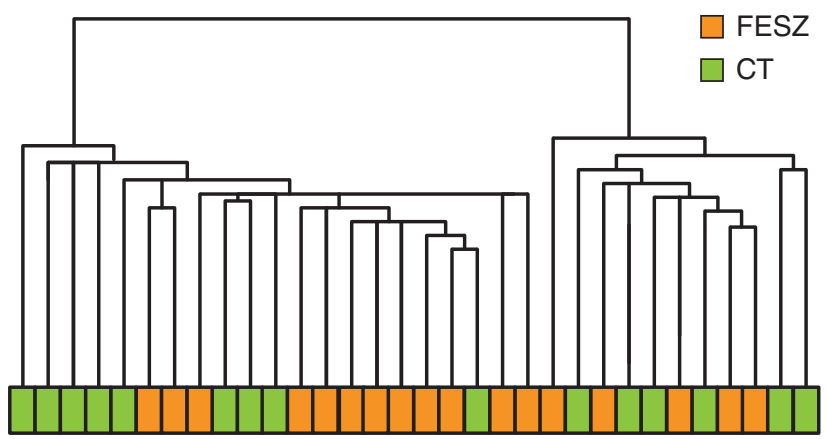

Figure 1 Unbiased hierarchical cluster analysis of 32 subjects based on the DNA methylation status of all probes. Cluster analysis did not discriminate between FESZ patients (orange boxes) and CTs (green boxes).
(Figure $2 \mathrm{~b}, P<9.3 \times 10^{-35}, \chi^{2}$ - test), suggesting significant hypomethylation of $\mathrm{CpG}$ islands in the FESZ patients.

The $603 \mathrm{CpG}$ sites covered 589 distinctive genes and were located mainly in promoter regions (Supplementary Table 1). Fourteen pairs of CpG sites covered the same genes. A top-ranked gene identified by SAM was CLDN12, which encodes a component of tight junction strands. The average beta-value difference between the FESZ and CT groups was 0.026 . The maximum beta-value difference between the two groups was 0.084 at the CpG site (cg17607973) in BCDIN3. This gene encodes a posttranslational protein modification enzyme and has not been reported in previous molecular genetics studies of schizophrenia. The interindividual beta-value differences for CLDN12 and BCDIN3 are shown in Figure 2c. The beta-value differences for the other genes discussed in this study are shown in Supplementary Figure 2.

Gene ontology analysis of the 589 genes revealed that genes related to the nuclear lumen, to nucleotide binding and to transcription factor binding were significantly enriched (Table 3).

We also analyzed correlations between clinical indicators (PANSS score (Total scale, Positive scale, Negative scale and General Psychopathology scale), GAF, DUP, age at disease onset and age at psychosis onset) and the beta-values of the 603 CpG sites (589 genes). Significant correlations are listed in Supplementary Table 2. ANKS3, which is related to the promising schizophrenia candidate gene ANK3, was negatively correlated with total PANSS score and

Table 2 Comparison of beta-values (average \pm s.d.) in FESZ patients and CTs

\begin{tabular}{lcccc}
\hline Location relative to CpG islands & CpG site count & Proportion (\%) & Beta-value in FESZ patients & Beta-value in CTs \\
\hline All CpG sites & 27578 & 100 & $0.351 \pm 0.003$ & $0.356 \pm 0.003$ \\
Inside CpG islands & 20006 & 72.5 & $0.271 \pm 0.186$ & $0.279 \pm 0.184$ \\
Outside CpG islands & 7572 & 27.5 & $0.564 \pm 0.158$ & $0.08 \times 10^{-5}$ \\
\hline
\end{tabular}

Abbreviations: CTs, controls; FESZ, first-episode schizophrenia.

a

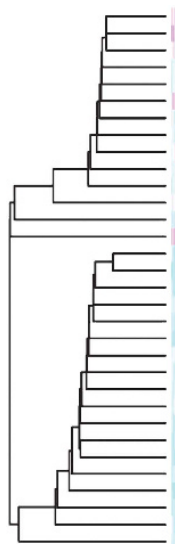

b
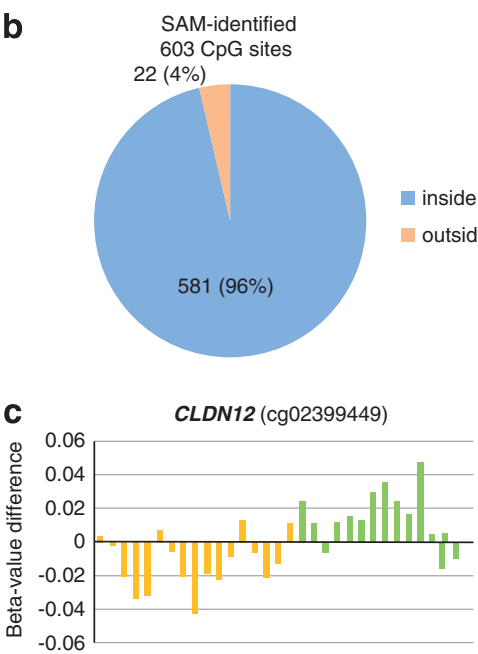

27,578 CpG sites in the Illumina Infinium HumanMethylation27
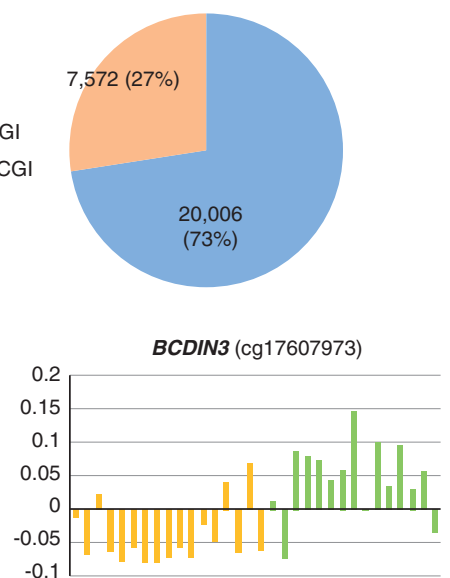

Figure 2 Differentially methylated CpG sites in FESZ patients. (a) Hierarchical cluster analysis of 603 SAM-identified CpG sites. Cluster analysis discriminated between FESZ patients (orange boxes) and CTs (green boxes). The gradation from blue to pink indicates the gradation from low to high standardized beta-values. (b) The 603 SAM-identified CpG sites were enriched inside CpG islands. The left panel shows the proportion of the 603 SAMidentified CpG sites by their location relative to CpG islands. The right panel shows the proportion of all CpG sites in the Illumina Infinium HumanMethylation27 BeadChip Kit by the location relative to CpG islands. CGI, CpG island. (c) Interindividual beta-value differences for CLDN12 and $B C D I N 3$. The $y$ axis is the difference from the average beta-value. 
Table 3 Gene ontology analysis of 589 SAM-identified genes $(\boldsymbol{P}<0.001)$

\begin{tabular}{|c|c|c|c|}
\hline Category & Term & Gene count & $\mathrm{P}$-value \\
\hline Cellular component & Membrane-enclosed lumen & 88 & $8.89 \times 10^{-7}$ \\
\hline Cellular component & Organelle lumen & 86 & $1.44 \times 10^{-6}$ \\
\hline Cellular component & Nucleoplasm & 51 & $1.92 \times 10^{-6}$ \\
\hline Cellular component & Intracellular organelle Iumen & 84 & $2.12 \times 10^{-6}$ \\
\hline Cellular component & Nuclear lumen & 67 & $6.45 \times 10^{-5}$ \\
\hline Biological process & Protein targeting & 18 & $2.31 \times 10^{-4}$ \\
\hline Biological process & Intracellular transport & 37 & $2.82 \times 10^{-4}$ \\
\hline Molecular function & Nucleotide binding & 93 & $4.27 \times 10^{-4}$ \\
\hline Molecular function & Transcription activator activity & 26 & $4.94 \times 10^{-4}$ \\
\hline Cellular component & Nucleoplasm part & 31 & $5.59 \times 10^{-4}$ \\
\hline Cellular component & Cytosol & 59 & $5.92 \times 10^{-4}$ \\
\hline Molecular function & Transcription factor binding & 30 & $6.10 \times 10^{-4}$ \\
\hline Biological process & Small GTPase mediated signal transduction & 21 & $7.78 \times 10^{-4}$ \\
\hline Biological process & Mitochondrion organization & 13 & $8.23 \times 10^{-4}$ \\
\hline
\end{tabular}

Abbreviation: SAM, Significance Analysis of Microarray.

positively correlated with GAF. TRA2A was negatively correlated with total PANSS score and positively correlated with age at disease onset, both of which indicate the severity of schizophrenia. CLDN12, a topranked differentially methylated gene as indicated by SAM, was negatively correlated with DUP (Figure 3).

\section{SAM analysis of male and female subjects}

Given that male and female schizophrenia patients have different clinical courses (for example, female schizophrenia patients have a later onset and better treatment response ${ }^{31}$ ), we separately compared the DNA methylation statuses of 21 male subjects (11 FESZ patients and $10 \mathrm{CTs}$ ) and 12 female subjects (7 FESZ patients and $5 \mathrm{CTs}$ ). We first performed cluster analysis for male and female subjects, separately. However, neither analysis discriminated between the FESZ patients and CTs (data not shown). We then performed SAM analysis separately and found that 1352 genes (1429 CpG sites) in males and 172 genes (173 CpG sites) in females had significantly different DNA methylation statuses. Only 32 genes were found in common (Supplementary Figure 3). Among these 32 genes, 27 genes overlapped with the 589 genes identified in whole samples (Supplementary Table 1). Note that the total number of overlapping CpG sites was 23, covering 23 genes. The remaining nine genes were covered by different $\mathrm{CpG}$ sites in male and female subjects.

Gene ontology analysis revealed that the genes related to transcription and to the nuclear lumen were enriched in the common 32 genes (Supplementary Table 3). In particular, the enrichment of nuclearlumen-related genes was concordant with the results of gene ontology analysis of whole samples (Table 3). The gene ontology of 140 femalespecific genes revealed characteristic enrichment of mitochondrionrelated genes $(P=0.0004, \mathrm{FDR}=0.6 \%$; Supplementary Table 4$)$. The results of gene ontology analysis of 1320 male-specific genes resembled the results of gene ontology analysis of whole samples (Table 3) (data not shown).

\section{Comparison with previous studies}

Previous studies of peripheral tissue samples indicated DNA methyla-

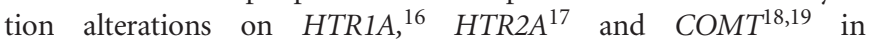
schizophrenia patients. In our study, SAM identified HTR1E and COMTD1 among the 589 genes (Supplementary Table 1), which are functionally related to HTR1A, HTR2A and COMT. However, HTR2A and COMT were not differentially methylated in SAM analysis, and HTR1A was not covered in this assay.

Dempster et al. ${ }^{20}$ reported that monozygotic twins discordant for psychosis show characteristic DNA methylation differences in peripheral blood samples. These investigators also used the Infinium HumanMethylation27 BeadChip Kit that we used in this study. Among the 100 top-ranked schizophrenia-associated genes in the study of Dempster et al. ${ }^{20}$ and the 589 genes we identified, there was one common gene, ADAMTS3. Both studies showed hypomethylation of ADAMTS3 (Supplementary Figure 2). Cluster analysis of 100 top-ranked schizophrenia-associated genes in the study of Dempster et al. ${ }^{20}$ with the beta-values of our data did not discriminate between the FESZ patients and the CTs (data not shown).

\section{DISCUSSION}

Although cluster analysis of the beta-values of all the CpG sites did not discriminate between the FESZ patients and the CTs, we identified global hypomethylation in the FESZ patients. This result is consistent with previous results, indicating that peripheral leukocytes are globally hypomethylated in schizophrenia patients, ${ }^{19,32}$ although another research group reported no difference between schizophrenia patients and CTs. ${ }^{33}$ Importantly, DNA methylation levels were different between $\mathrm{CpG}$ sites inside and outside CpG islands in our study. The observation that the CpG sites inside CpG islands showed significant hypomethylation in FESZ patients implies that alterations in DNA methylation have functional significance related to gene regulation. We also identified site-specific DNA methylation changes in the FESZ patients. Significantly, hypomethylated genes in FESZ patients included genes related to the nuclear lumen, to nucleotide binding and to transcription factor binding. Among these three groups, genes related to the nuclear lumen showed altered methylation status in both genders, suggesting the possibility of uncharacterized deficits in the peripheral blood cells of FESZ patients. In addition, HTR1E and COMTD1 were among the SAM-identified genes. These two genes are functionally related to $H T R 1 A,{ }^{16} H T R 2 A^{17}$ and $C O M T,{ }^{18,19}$ which are differentially methylated in the peripheral tissues, according to previous reports. Our results did not seem to overlap with the results of Dempster et al. ${ }^{20}$ even though we used the same platform. This lack of overlap may be partly attributable to differences in the disease stages of the study subjects. However, 

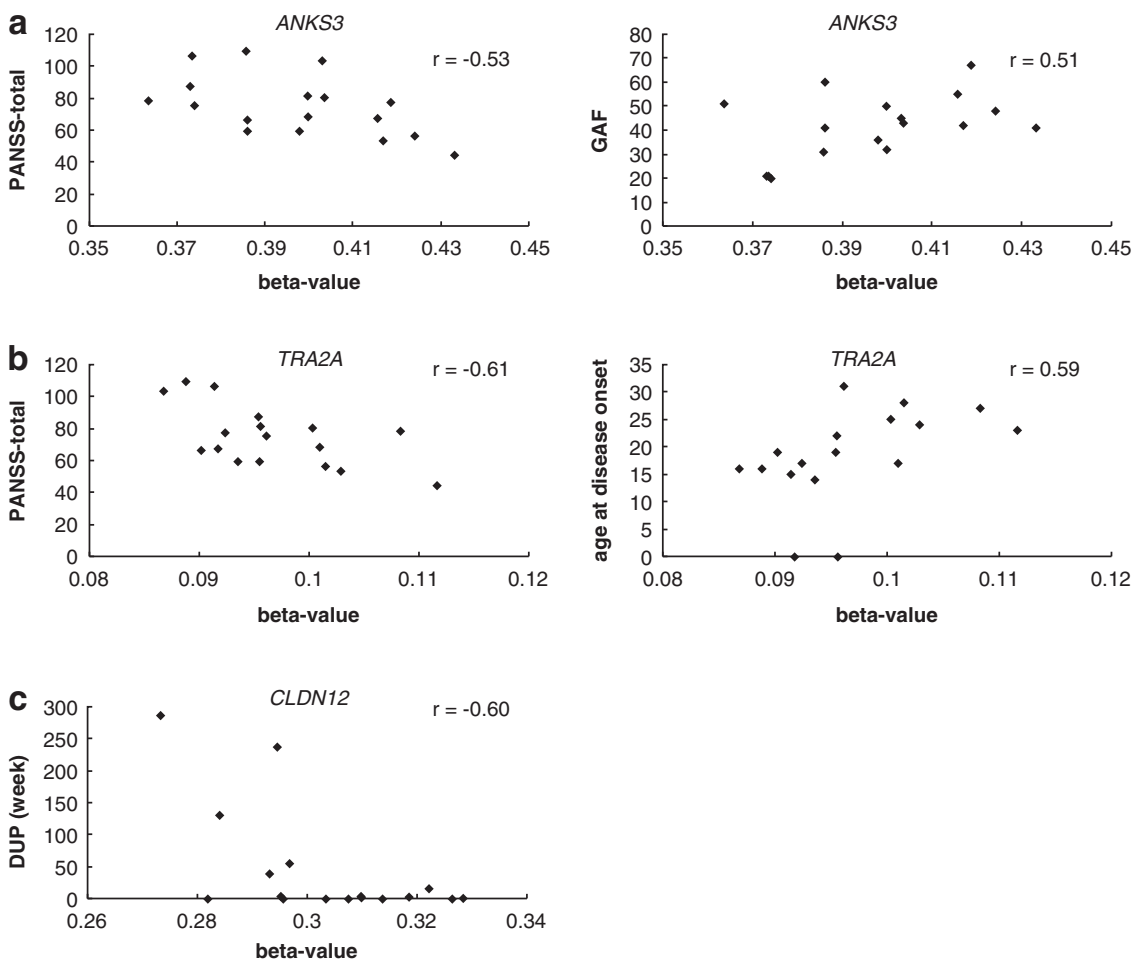

Figure 3 Correlation between beta-values of selected genes and clinical indicators (total PANSS score, GAF, DUP and age at disease onset). The correlation coefficients are shown in Supplementary Table S2. (a) The beta-value of ANKS3 was negatively correlated with total PANSS score and positively correlated with GAF. (b) The beta-value of TRA2A was negatively correlated with total PANSS score and positively correlated with age at disease onset. (c) The betavalue of CLDN12 was negatively correlated with DUP.

ADAMTS3, a disintegrin and metalloproteinase with thrombospondin motifs 3, showed consistent hypomethylation both in the results of Dempster et al. and our results. Although the functional significance of ADAMTS3 remains unclear, a recent exome study identified de novo mutation of this gene in a patient with sporadic schizophrenia. ${ }^{34}$ Our results also showed slight overlap with the study of Kinoshita et al. using Infinium HumanMethylation450 BeadChip Kit (Illumina). ${ }^{35}$ They mainly recruited first-onset schizophrenia patients, who had similar profiles to the subjects in our study. Little overlap between two previous results and our results may be due to small sample size in these studies and the heterogeneity of schizophrenia. Further studies with larger sample size and clinically homogeneous subjects will be needed.

We also observed hypomethylation of SLC6A3, NEUROD2, NEUROD4 and HDAC11 (Supplementary Figure 2). SLC6A3 encodes a dopamine transporter and is reportedly related to mental disorders including attention-deficit hyperactivity disorder and dependence on alcohol or cocaine. ${ }^{36}$ NEUROD2 and NEUROD4 are crucial in neuronal differentiation. ${ }^{37,38}$ HDAC11 encodes a histone deacetylase and has an important role in epigenetic modifications. The methylation levels of these interesting genes related to brain function including HTR1E and COMTD1, as well as other important genes, need validation and further investigation in detail.

In our study, the difference of beta-values between FESZ patients and CTs was not large enough to conclude the physiological meaning of methylation difference. The average $\Delta$ beta-value of 0.026 in 603 SAM-identified CpG sites may be regarded as relatively small, but previous studies with the same platform reported $\Delta$ beta-values of 0.10 at most. ${ }^{20,39,40}$ In particular, Dempster et al. ${ }^{20}$ reported a $\Delta$ beta-value of $\sim 0.05$ with the same platform in peripheral blood cells from schizophrenia patients. We did not examine the expression levels of each investigated genes in relation to methylation differences, but further examination will be needed to assess the physiological meanings of these small differences.

We identified several genes whose beta-values were correlated with clinical indicators. Previous studies reported that longer DUP is associated with poorer prognosis and that early intervention is needed for the FESZ patients to shorten DUP. ${ }^{41}$ The correlated genes with DUP are potential biomarkers not only for prognosis but also for the indication of early intervention, which are needed in clinical practice. For example, the hypomethylation of CLDN12, which was correlated with long DUP in our study, is the potential signal for early intervention. The correlated genes with PANSS or GAF are also potential biomarkers for the disease severity. For example, the hypomethylation of $A N K S 3$, which was correlated with high PANSS score and low GAF, is the potential indicator of disease severity. These correlations between hypomethylation and poor prognosis or disease severity are consistent with our results that the methylation levels in the FESZ patients were lower than those in CTs. In addition, individual PANSS-correlated genes may explain the respective components of schizophrenia etiology such as positive symptoms and negative symptoms.

Note that only a small number of genes showed overlapping methylation changes in both genders, suggesting that differential epigenetic changes may reflect gender-specific pathophysiology. In particular, female patients tended to have epigenetic alterations 
in mitochondrion-related genes, which should be validated in an independent study of large sample sets.

The sample size in our study is relatively small, because we strictly selected the patients according to the rigid criteria described in Materials and methods and we excluded subjects with a family history of an axis I mental disorder in their first-degree relatives from the $\mathrm{CTs}$, to preclude the influence of genetic factors as much as possible. Therefore, studies with larger sample size are required to validate our results. In addition, the effect of psychotropic drugs on the epigenome cannot be ruled out, although our subjects had been medicated for only a short time (10.6 \pm 9.1 weeks). Future studies are needed to elucidate the effects of psychotropic drugs on epigenome.

In conclusion, our study indicated that peripheral blood samples from FESZ patients showed specific epigenetic alterations. Our results indicate that nuclear-lumen-related genes and other functionally important genes including HTR1E, COMTD1 and SLC6A3 show epigenetic alterations in FESZ patients.

\section{CONFLICT OF INTEREST}

$\mathrm{MN}, \mathrm{MB}$ and KI belong to the Department of Molecular Psychiatry, which is an endowment department by Dainippon Sumitomo Pharma, Yoshitomiyakuhin and Astellas Pharma. They had no role in study design, data collection and analysis, decision to publish or preparation of the manuscript. The remaining authors declare no conflict of interest.

\section{ACKNOWLEDGEMENTS}

We thank the members of the Integrative Neuroimaging studies for Schizophrenia Targeting Early intervention and Prevention (IN-STEP) research team at the University of Tokyo Hospital for their assistance with this project. We thank Tsukasa Sasaki for his support to sample collection. We thank Junko Ueda and Fumiko Sunaga for their technical assistance. We gratefully acknowledge all the participants in this study. This study was partly funded by the Development of Biomarker Candidates for Social Behavior project carried out under the Strategic Research Program for Brain Sciences by the Ministry of Education, Culture, Sports, Science and Technology in Japan.

1 Gershon, E. S., Alliey-Rodriguez, N. \& Liu, C. After GWAS: searching for genetic risk for schizophrenia and bipolar disorder. Am. J. Psychiatry. 168, 253-256 (2011).

2 Stefansson, H., Ophoff, R. A., Steinberg, S., Andreassen, O. A., Cichon, S., Rujescu, D. et al. Common variants conferring risk of schizophrenia. Nature. 460, 744-747 (2009).

3 O'Donovan, M. C., Craddock, N., Norton, N., Williams, H., Peirce, T., Moskvina, V. et al. Identification of loci associated with schizophrenia by genome-wide association and follow-up. Nat. Genet. 40, 1053-1055 (2008).

4 Ripke, S., Sanders, A. R., Kendler, K. S., Levinson, D. F., Sklar, P., Holmans, P. A. et al. Genome-wide association study identifies five new schizophrenia loci. Nat. Genet. $\mathbf{4 3}$ 969-976 (2011).

5 Sharma, R. P., Grayson, D. R. \& Gavin, D. P. Histone deactylase 1 expression is increased in the prefrontal cortex of schizophrenia subjects: analysis of the National Brain Databank microarray collection. Schizophr. Res. 98, 111-117 (2008).

6 Grayson, D. R., Jia, X., Chen, Y., Sharma, R. P., Mitchell, C. P., Guidotti, A. et al. Reelin promoter hypermethylation in schizophrenia. Proc. Natl Acad. Sci. USA. 102, 9341-9346 (2005)

7 Abdolmaleky, H. M., Cheng, K. H., Russo, A., Smith, C. L., Faraone, S. V., Wilcox, M. et al. Hypermethylation of the reelin (RELN) promoter in the brain of schizophrenic patients: a preliminary report. Am. J. Med. Genet. B Neuropsychiatr. Genet. 134B, 60-66 (2005)

8 Abdolmaleky, H. M., Cheng, K. H., Faraone, S. V., Wilcox, M., Glatt, S. J., Gao, F. et al. Hypomethylation of MB-COMT promoter is a major risk factor for schizophrenia and bipolar disorder. Hum. Mol. Genet. 15, 3132-3145 (2006)

9 Iwamoto, K., Bundo, M., Yamada, K., Takao, H., Iwayama-Shigeno, Y., Yoshikawa, T. et al. DNA methylation status of SOX10 correlates with its downregulation and oligodendrocyte dysfunction in schizophrenia. J. Neurosci. 25, 5376-5381 (2005).

10 Tolosa, A., Sanjuan, J., Dagnall, A. M., Molto, M. D., Herrero, N. \& de Frutos, R. FOXP2 gene and language impairment in schizophrenia: association and epigenetic studies. BMC Med. Genet. 11, 114 (2010)
11 Pun, F. W., Zhao, C., Lo, W. S., Ng, S. K., Tsang, S. Y., Nimgaonkar, V. et al. Imprinting in the schizophrenia candidate gene GABRB2 encoding GABA(A) receptor beta(2) subunit. Mol. Psychiatry. 16, 557-568 (2011)

12 Abdolmaleky, H. M., Yaqubi, S., Papageorgis, P., Lambert, A. W., Ozturk, S., Sivaraman, V. et al. Epigenetic dysregulation of HTR2A in the brain of patients with schizophrenia and bipolar disorder. Schizophr. Res. 129, 183-190 (2011).

13 Dempster, E. L., Mill, J., Craig, I. W. \& Collier, D. A. The quantification of COMT mRNA in post mortem cerebellum tissue: diagnosis, genotype, methylation and expression. BMC Med. Genet. 7, 10 (2006).

14 Mill, J., Tang, T., Kaminsky, Z., Khare, T., Yazdanpanah, S., Bouchard, L. et al. Epigenomic profiling reveals DNA-methylation changes associated with major psychosis. Am. J. Hum. Genet. 82, 696-711 (2008).

15 Tochigi, M., Iwamoto, K., Bundo, M., Komori, A., Sasaki, T., Kato, N. et al. Methylation status of the reelin promoter region in the brain of schizophrenic patients. Biol. Psychiatry. 63, 530-533 (2008).

16 Carrard, A., Salzmann, A., Malafosse, A. \& Karege, F. Increased DNA methylation status of the serotonin receptor 5HTR1A gene promoter in schizophrenia and bipolar disorder. J. Affect. Disord. 132, 450-453 (2011).

17 Ghadirivasfi, M. Nohesara, S., Ahmadkhaniha, H. R., Eskandari, M. R., Mostafavi, S., Thiagalingam, S. et al. Hypomethylation of the serotonin receptor type-2A Gene (HTR2A) at T102C polymorphic site in DNA derived from the saliva of patients with schizophrenia and bipolar disorder. Am. J. Med. Genet. B Neuropsychiatr. Genet. 156, 536-545 (2011).

18 Nohesara, S., Ghadirivasfi, M., Mostafavi, S., Eskandari, M. R., Ahmadkhaniha, H., Thiagalingam, $\mathrm{S}$. et al. DNA hypomethylation of MB-COMT promoter in the DNA derived from saliva in schizophrenia and bipolar disorder. J. Psychiatr. Res. 45, 1432-1438 (2011)

19 Melas, P. A., Rogdaki, M., Osby, U., Schalling, M., Lavebratt, C. \& Ekstrom, T. J. Epigenetic aberrations in leukocytes of patients with schizophrenia: association of global DNA methylation with antipsychotic drug treatment and disease onset. FASEB J. 26, 2712-2718 (2012).

20 Dempster, E. L., Pidsley, R., Schalkwyk, L. C., Owens, S., Georgiades, A., Kane, F. et al. Disease-associated epigenetic changes in monozygotic twins discordant for schizophrenia and bipolar disorder. Hum. Mol. Genet. 20, 4786-4796 (2011).

21 Murphy, B. C., O'Reilly, R. L. \& Singh, S. M. Site-specific cytosine methylation in S-COMT promoter in 31 brain regions with implications for studies involving schizophrenia. Am. J. Med. Genet. B Neuropsychiatr. Genet. 133B, 37-42 (2005).

22 Sugawara, H., Iwamoto, K., Bundo, M., Ueda, J., Miyauchi, T., Komori, A. et al. Hypermethylation of serotonin transporter gene in bipolar disorder detected by epigenome analysis of discordant monozygotic twins. Transl. Psychiatry. 1, e24 (2011)

23 Kaminsky, Z., Tochigi, M., Jia, P., Pal, M., Mill, J., Kwan, A. et al. A multi-tissue analysis identifies HLA complex group 9 gene methylation differences in bipolar disorder. Mol. Psychiatry. 17, 728-740 (2012).

24 McGlashan, T. H., Miller, T. J. \& Woods, S. W. Structured Interview for Prodromal Syndromes (version 3.0). (PRIME Research Clinic, Yale School of Medicine, New Haven, 2001).

25 Bibikova, M., Le, J., Barnes, B., Saedinia-Melnyk, S., Zhou, L., Shen, R. et al. Genome-wide DNA methylation profiling using Infinium(R) assay. Epigenomics. 1, 177-200 (2009).

26 Lucas, A. \& Jasson, S. Using amap and ctc packages for huge clustering. $R$ News. 6 , 58-60 (2006).

27 Tusher, V. G., Tibshirani, R. \& Chu, G. Significance analysis of microarrays applied to the ionizing radiation response. Proc. Natl Acad. Sci. USA. 98, 5116-5121 (2001).

28 Efron, B. \& Tibshirani, R. Empirical bayes methods and false discovery rates for microarrays. Genet. Epidemiol. 23, 70-86 (2002).

29 Huang da, W., Sherman, B. T. \& Lempicki, R. A. Bioinformatics enrichment tools: paths toward the comprehensive functional analysis of large gene lists. Nucleic Acids Res. 37, 1-13 (2009)

30 Huang da, W., Sherman, B. T. \& Lempicki, R. A. Systematic and integrative analysis of large gene lists using DAVID bioinformatics resources. Nat. Protoc. 4, 44-57 (2009).

31 Szymanski, S., Lieberman, J. A., Alvir, J. M., Mayerhoff, D., Loebel, A., Geisler, S. et al Gender differences in onset of illness, treatment response, course, and biologic indexes in first-episode schizophrenic patients. Am. J. Psychiatry. 152, 698-703 (1995)

32 Shimabukuro, M., Sasaki, T., Imamura, A., Tsujita, T., Fuke, C., Umekage, T. et al. Global hypomethylation of peripheral leukocyte DNA in male patients with schizophrenia: a potential link between epigenetics and schizophrenia. J. Psychiatr. Res. 41, 1042-1046 (2007).

33 Bromberg, A., Levine, J., Nemetz, B., Belmaker, R. H. \& Agam, G. No association between global leukocyte DNA methylation and homocysteine levels in schizophrenia patients. Schizophr. Res. 101, 50-57 (2008).

34 Xu, B., Roos, J. L., Dexheimer, P., Boone, B., Plummer, B., Levy, S et al. Exome sequencing supports a de novo mutational paradigm for schizophrenia. Nat. Genet. 43 864-868 (2011).

35 Kinoshita, M., Numata, S., Tajima, A., Shimodera, S., Ono, S., Imamura, A. et al. DNA methylation signatures of peripheral leukocytes in schizophrenia. Neuromol. Med. (e-pub ahead of print 9 September 2012; doi:10.1007/s12017-12012-18198 12016).

36 Vandenbergh, D. J., Thompson, M. D., Cook, E. H., Bendahhou, E., Nguyen, T., Krasowski, M. D. et al. Human dopamine transporter gene: coding region conservation 
among normal, Tourette's disorder, alcohol dependence and attention-deficit hyperactivity disorder populations. Mol. Psychiatry. 5, 283-292 (2000).

37 Lin, C. H., Hansen, S., Wang, Z., Storm, D. R., Tapscott, S. J. \& Olson, J. M. The dosage of the neuroD2 transcription factor regulates amygdala development and emotional learning. Proc. Natl Acad. Sci. USA. 102, 14877-14882 (2005).

38 Pinto, L., Drechsel, D., Schmid, M. T., Ninkovic, J., Irmler, M., Brill, M. S. et al. AP2gamma regulates basal progenitor fate in a region- and layer-specific manner in the developing cortex. Nat. Neurosci. 12, 1229-1237 (2009).
39 Banister, C. E., Koestler, D. C., Maccani, M. A., Padbury, J. F., Houseman, E. A. \& Marsit, C. J. Infant growth restriction is associated with distinct patterns of DNA methylation in human placentas. Epigenetics. 6, 920-927 (2011).

40 Bell, C. G., Teschendorff, A. E., Rakyan, V. K., Maxwell, A. P., Beck, S. \& Savage, D. A. Genome-wide DNA methylation analysis for diabetic nephropathy in type 1 diabetes mellitus. BMC Med. Genomics. 3, 33 (2010).

41 Marshall, M., Lewis, S., Lockwood, A., Drake, R., Jones, P. \& Croudace, T. Association between duration of untreated psychosis and outcome in cohorts of first-episode patients: a systematic review. Arch. Gen. Psychiatry. 62, 975-983 (2005).

Supplementary Information accompanies the paper on Journal of Human Genetics website (http://www.nature.com/jhg) 